\title{
A Discrete-Time American Put Option Model with Fuzziness of Stock Prices
}

\author{
Y. Yoshida (yoshida@kitakyushu.ac.jp) \\ The University of Kitakyushu, Japan \\ M. Yasuda, J. Nakagami and M. Kurano \\ Chiba University, Japan
}

\begin{abstract}
To solve a mathematical model for American put option with uncertainty, we utilize two essentials, i.e., a $\lambda$-weighting function and a mean value of fuzzy random variables simultaneously. Estimation of randomness and fuzziness as uncertainty should be important when we deal with a reasonable and natural model extended from the original optimization/decision making. Three kinds of mean values by fuzzy measures, which are based on Possibility, Necessity and Credibility, are demonstrated particularly. We consider the optimal expected price of the American put option by dynamic programming under a reasonable assumption. A numerical example is given to illustrate our idea.
\end{abstract}

Keywords: Decision making with uncertainty, American put option, fuzzy stochastic process, optimal stopping, fuzzy measures, $\lambda$-weighting functions, financial engineering.

\section{INTRODUCTION}

The option pricing for stocks plays an important role in stock markets. Approaches with fuzzy logic to European options are discussed by some authors(C.Carlsson and R.Fullér [2], M.R.Simonelli [13], Y.Yoshida [18], Z.Zmeškal[20]). This paper deals with a discrete-time mathematical model for American put option with uncertainty of stock prices. It is not easy in general to calculate American put option with an expiration date in continuous-time systems, and the study of the discretetime case is one of the most important approaches to investigate the continuous-time model through approximation. Mathematical modeling of stochastic systems in optimization/decision-making has many applications to engineering, economics, etc.. One of the conditions that stochastic modeling works successfully is stability of systems. If the systems are unstable when the models are applied actually, losses/errors, which cannot be explained by only probabilistic elements, sometimes occur between the decision maker's expected price and the actual price. On selling and buying stocks by means of internet in financial markets when stock prices change radically, the loss from time lag through internet etc. might be more huge. This kind of loss is not only a problem

(C) 2005 Kluwer Academic Publishers. Printed in the Netherlands. 
arising from probabilistic sense where something occurs or not, and it is difficult to formulate them by only probabilistic theory.

In this paper, probability is applied as the uncertainty such that something occurs or not with probability, and fuzziness is applied as the uncertainty such that we cannot specify the exact prices because of a lack of knowledge regarding the present stock market. By introducing fuzziness to stochastic processes in optimization/decision-making, we consider a new model with uncertainty of both randomness and fuzziness, which is a reasonable and natural extension of the original stochastic process. The optimal stopping problem for a sequence of random variables has a long history and has been studied by many authors in financial engineering(Elliott and Kopp [3], Pliska [9], Ross [11] and so on).

We need to discuss the optimal stopping problem with randomness and fuzziness as uncertainty since the American put option model are represented by an optimal stopping problem in stochastic processes. In order to describe an optimal stopping model with fuzziness, we need to extend real-valued random variables in the classical probability theory to 'fuzzy random variables' which are fuzzy-number valued random variables. Fuzzy random variables were first studied by Puri and Ralescu [10] and have been studied by many authors. It is known that the fuzzy random variable is one of the successful hybrid notions of randomness and fuzziness.

In the next section, we introduce a fuzzy stochastic process by fuzzy random variables to define prices in American put option with uncertainty. The prices are called 'fuzzy prices' in this paper. Extending the idea in Yoshida et al. [17], we introduce mean values of a fuzzy number defined by fuzzy measures and $\lambda$-weighting functions. This paper evaluates the randomness and fuzziness in fuzzy stochastic processes by the probabilistic expectation and the mean values defined by fuzzy measures and $\lambda$-weighting functions. The mean values are demonstrated particularly in three kinds of important fuzzy measures: possibility measure, necessity measure and credibility measure. In Section 3, American put option model with uncertainty is formulated and fuzzy prices of the American option are evaluated by the probabilistic expectation and mean values defined by fuzzy measures and $\lambda$-weighting functions from the viewpoint of Yoshida et al. [16]. In Section 4 , an optimality equation for the optimal fuzzy price is derived by dynamic programming under a reasonable assumption. Next, we consider the optimal expected price of the American put option and writer's (seller's) optimal expected prices. Further, an optimal exercise time is given for the American put option. Finally, in the last section, a numerical example is given to illustrate our idea. 


\section{FuZZY StochastiC PROCESSES}

First we give some mathematical notations regarding fuzzy numbers. Let $(\Omega, \mathcal{M}, P)$ be a probability space, where $\mathcal{M}$ is a $\sigma$-field of $\Omega$ and $P$ is a non-atomic probability measure. $\mathbb{R}$ denotes the set of all real numbers, and let $\mathcal{B}(\mathbb{R})$ and $\mathcal{C}(\mathbb{R})$ be the Borel $\sigma$-field of $\mathbb{R}$ and the set of all non-empty bounded closed intervals respectively. A 'fuzzy number' is denoted by its membership function $\tilde{a}: \mathbb{R} \mapsto[0,1]$ which is normal, upper-semicontinuous, fuzzy convex and has a compact support. $\mathcal{R}$ denotes the set of all fuzzy numbers, and $\mathcal{R}_{c}$ is the set of fuzzy numbers with continuous membership functions. Refer to Zadeh [19] regarding fuzzy set theory.

Fuzzy numbers with its corresponding membership functions are identified in this paper. The $\alpha$-cut of a fuzzy number $\tilde{a}(\in \mathcal{R})$ is given by $\tilde{a}_{\alpha}:=\{x \in \mathbb{R} \mid \tilde{a}(x) \geq \alpha\}(\alpha \in(0,1])$ and $\tilde{a}_{0}:=\operatorname{cl}\{x \in \mathbb{R} \mid \tilde{a}(x)>0\}$, where cl denotes the closure of an interval. In this paper, we write the closed intervals by $\tilde{a}_{\alpha}:=\left[\tilde{a}_{\alpha}^{-}, \tilde{a}_{\alpha}^{+}\right]$for $\alpha \in[0,1]$. Hence we introduce a partial order $\succeq$, so called the 'fuzzy max order', on fuzzy numbers $\mathcal{R}$ : Let $\tilde{a}, \tilde{b} \in \mathcal{R}$ be fuzzy numbers. $\tilde{a} \succeq \tilde{b}$ means that $\tilde{a}_{\alpha}^{-} \geq \tilde{b}_{\alpha}^{-}$and $\tilde{a}_{\alpha}^{+} \geq \tilde{b}_{\alpha}^{+}$ for all $\alpha \in[0,1]$. Then $(\mathcal{R}, \succeq)$ becomes a lattice. For fuzzy numbers $\tilde{a}, \tilde{b} \in \mathcal{R}$, we define the maximum $\tilde{a} \vee \tilde{b}$ with respect to the fuzzy max order $\succeq$ by the fuzzy number whose $\alpha$-cuts are

$$
(\tilde{a} \vee \tilde{b})_{\alpha}=\left[\max \left\{\tilde{a}_{\alpha}^{-}, \tilde{b}_{\alpha}^{-}\right\}, \max \left\{\tilde{a}_{\alpha}^{+}, \tilde{b}_{\alpha}^{+}\right\}\right], \quad \alpha \in[0,1] .
$$

An addition, a subtraction and a scalar multiplication for fuzzy numbers are defined as follows: For $\tilde{a}, \tilde{b} \in \mathcal{R}$ and $\mu \geq 0$, the addition and subtraction $\tilde{a} \pm \tilde{b}$ of $\tilde{a}$ and $\tilde{b}$ and the scalar multiplication $\mu \tilde{a}$ of $\mu$ and $\tilde{a}$ are fuzzy numbers given by

$$
\begin{aligned}
(\tilde{a}+\tilde{b})_{\alpha}:= & {\left[\tilde{a}_{\alpha}^{-}+\tilde{b}_{\alpha}^{-}, \tilde{a}_{\alpha}^{+}+\tilde{b}_{\alpha}^{+}\right], \quad(\tilde{a}-\tilde{b})_{\alpha}:=\left[\tilde{a}_{\alpha}^{-}-\tilde{b}_{\alpha}^{+}, \tilde{a}_{\alpha}^{+}-\tilde{b}_{\alpha}^{-}\right] } \\
& \text {and }(\mu \tilde{a})_{\alpha}:=\left[\mu \tilde{a}_{\alpha}^{-}, \mu \tilde{a}_{\alpha}^{+}\right] \quad \text { for } \alpha \in[0,1] .
\end{aligned}
$$

A fuzzy-number-valued map $\tilde{X}: \Omega \mapsto \mathcal{R}$ is called a 'fuzzy random variable' if the maps $\omega \mapsto \tilde{X}_{\alpha}^{-}(\omega)$ and $\omega \mapsto \tilde{X}_{\alpha}^{+}(\omega)$ are measurable for all $\alpha \in[0,1]$, where $\tilde{X}_{\alpha}(\omega)=\left[\tilde{X}_{\alpha}^{-}(\omega), \tilde{X}_{\alpha}^{+}(\omega)\right]=\{x \in \mathbb{R} \mid \tilde{X}(\omega)(x) \geq$ $\alpha\}$ (see [15]). Next we need to introduce expectations of fuzzy random variables in order to describe an optimal stopping model in the next section. A fuzzy random variable $\tilde{X}$ is called integrably bounded if both $\omega \mapsto \tilde{X}_{\alpha}^{-}(\omega)$ and $\omega \mapsto \tilde{X}_{\alpha}^{+}(\omega)$ are integrable for all $\alpha \in[0,1]$. Let $\tilde{X}$ be an integrably bounded fuzzy random variable. The expectation $E(\tilde{X})$ of the fuzzy random variable $\tilde{X}$ is defined by a fuzzy number (see [10])

$$
E(\tilde{X})(x):=\sup _{\alpha \in[0,1]} \min \left\{\alpha, 1_{E(\tilde{X})_{\alpha}}(x)\right\}, \quad x \in \mathbb{R},
$$


where $E(\tilde{X})_{\alpha}:=\left[\int_{\Omega} \tilde{X}_{\alpha}^{-}(\omega) \mathrm{d} P(\omega), \int_{\Omega} \tilde{X}_{\alpha}^{+}(\omega) \mathrm{d} P(\omega)\right](\alpha \in[0,1])$. We note that the map $\alpha \mapsto \int_{\Omega} \tilde{X}_{\alpha}^{ \pm}(\omega) \mathrm{d} P(\omega)$ is continuous by the monotone convergence theorem since $\alpha \mapsto \tilde{X}_{\alpha}^{ \pm}(\omega)$ is continuous for $\omega \in \Omega$. In the rest of this section, we introduce stopping times for fuzzy stochastic processes. Let $\mathbb{T}:=\{0,1,2, \cdots, T\}$ be the time space, where a positive integer $T$ is called an 'expiration date'. Let a 'fuzzy stochastic process' $\left\{\tilde{X}_{t}\right\}_{t=0}^{T}$ be a sequence of integrably bounded fuzzy random variables such that $E\left(\max _{t \in \mathbb{T}} \tilde{X}_{t, 0}^{+}\right)<\infty$, where $\tilde{X}_{t, 0}^{+}(\omega)$ is the rightend of the 0 -cut of the fuzzy number $\tilde{X}_{t}(\omega)$. For $t \in \mathbb{T}, \mathcal{M}_{t}$ denotes the smallest $\sigma$-field on $\Omega$ generated by all random variables $\tilde{X}_{s, \alpha}^{-}$and $\tilde{X}_{s, \alpha}^{+}$ $(s=0,1,2, \cdots, t ; \alpha \in[0,1])$. We call $\left(\tilde{X}_{t}, \mathcal{M}_{t}\right)_{t=0}^{\infty}$ a 'fuzzy stochastic process'. A map $\tau: \Omega \mapsto \mathbb{T}$ is called a 'stopping time' if $\{\omega \in \Omega \mid$ $\tau(\omega)=t\} \in \mathcal{M}_{t}$ for all $t=0,1,2, \cdots, T$. Then, the following lemma is trivial from the definitions ([16]).

Lemma 2.1. Let $\tau$ be a stopping time. We define

$$
\tilde{X}_{\tau}(\omega):=\tilde{X}_{t}(\omega) \quad \text { if } \tau(\omega)=t \quad \text { for } t=0,1,2, \cdots, T \text { and } \omega \in \Omega .
$$

Then, $\tilde{X}_{\tau}$ is a fuzzy random variable.

Next we consider the evaluation of fuzzy random variables. Fuzzy random variables have two kinds of uncertainty (randomness and fuzziness). In this paper, the randomness is evaluated by the probabilistic expectation, and the fuzziness is evaluated by $\lambda$-weighting functions and fuzzy measures. Let $g: \mathcal{C}(\mathbb{R}) \mapsto \mathbb{R}$ be a map such that

$$
g([x, y]):=\lambda x+(1-\lambda) y, \quad[x, y] \in \mathcal{C}(\mathbb{R}),
$$

where $\lambda$ is a constant satisfying $0 \leq \lambda \leq 1$. This scalarization is used for the estimation of fuzzy numbers, and $\lambda$ is called a 'pessimisticoptimistic index' and means the pessimistic degree in decision making (Fortemps and Roubens [4]). We call $g$ a ' $\lambda$-weighting function'.

Definition 2.1 (Wang and Klir [14]). A map $M: \mathcal{B} \mapsto[0,1]$ is called a 'fuzzy measure' on $\mathcal{B}$ if $M$ satisfies the following (M.i), (M.ii) and (M.iii) (or (M.i), (M.ii) and (M.iv)):

(M.i) $M(\emptyset)=0$ and $M(\mathbb{R})=1$;

(M.ii) $M\left(I_{1}\right) \leq M\left(I_{2}\right)$ holds for $I_{1}, I_{2} \in \mathcal{B}$ satisfying $I_{1} \subset I_{2}$;

(M.iii) $M\left(\bigcup_{n=0}^{\infty} I_{n}\right)=\lim _{n \rightarrow \infty} M\left(I_{n}\right)$ holds for $\left\{I_{n}\right\}_{n=0}^{\infty} \subset \mathcal{B}$ satisfying $I_{n} \subset I_{n+1}(n=0,1,2, \cdots)$;

(M.iv) $M\left(\bigcap_{n=0}^{\infty} I_{n}\right)=\lim _{n \rightarrow \infty} M\left(I_{n}\right)$ holds for $\left\{I_{n}\right\}_{n=0}^{\infty} \subset \mathcal{B}$ satisfying $I_{n} \supset I_{n+1}(n=0,1,2, \cdots)$. 
Extending the idea in Yoshida et al. [17], we introduce mean values of a fuzzy number as follows: Using fuzzy measures $M$ and $\lambda$-weighting functions $g$, we define mean values of a fuzzy number $\tilde{a} \in \mathcal{R}$ by

$$
\tilde{E}(\tilde{a})=\int_{0}^{1} M_{\tilde{a}}\left(\tilde{a}_{\alpha}\right) g\left(\tilde{a}_{\alpha}\right) \mathrm{d} \alpha / \int_{0}^{1} M_{\tilde{a}}\left(\tilde{a}_{\alpha}\right) \mathrm{d} \alpha,
$$

where $\tilde{a}_{\alpha}$ is the $\alpha$-cut of the fuzzy number $\tilde{a} . M_{\tilde{a}}$ is a fuzzy measure depending on the fuzzy number $\tilde{a},(2.4)$ is normalized by the values $M_{\tilde{a}}\left(\tilde{a}_{\alpha}\right)(\alpha \in[0,1])$, and $M_{\tilde{a}}\left(\tilde{a}_{\alpha}\right)$ means the confidence degree that the fuzzy number $\tilde{a}$ takes values at the interval $\tilde{a}_{\alpha}$ (see Example 2.1). Next, let $\tilde{a} \in \mathcal{R}$, and we define its mean value by

$$
\tilde{E}(\tilde{a}):=\lim _{n \rightarrow \infty} \tilde{E}\left(\tilde{a}^{n}\right),
$$

where $\left\{\tilde{a}^{n}\right\}_{n=1}^{\infty}\left(\subset \mathcal{R}_{c}\right)$ is a sequence of fuzzy numbers whose membership functions are continuous and satisfy $\tilde{a}^{n} \downarrow \tilde{a}$ pointwise as $n \rightarrow \infty$. If the limiting value (2.5') is independent of the selection of the sequences $\left\{\tilde{a}^{n}\right\}_{n=1}^{\infty} \subset \mathcal{R}_{c}$, we call (2.5') well-defined. In this paper, we deal with the case where the limiting value is well-defined (see Example 2.1).

Example 2.1. Let a fuzzy number $\tilde{a} \in \mathcal{R}_{c}$. An evaluation measure $M_{\tilde{a}}$ is called the 'possibility evaluation measure', the 'necessity evaluation measure' and the 'credibility evaluation measure' induced from the fuzzy number $\tilde{a}$ if it is given by the following (2.6) and (2.7) respectively:

$$
\begin{array}{rlrl}
M_{\tilde{a}}^{P}(I) & :=\sup _{x \in I} \tilde{a}(x), & I \in \mathcal{B} ; \\
M_{\tilde{a}}^{N}(I):=1-\sup _{x \notin I} \tilde{a}(x), & I \in \mathcal{B} ; \\
M_{\tilde{a}}^{C}(I):=\frac{1}{2}\left(\sup _{x \in I} \tilde{a}(x)+1-\sup _{x \notin I} \tilde{a}(x)\right), & I \in \mathcal{B} .
\end{array}
$$

We note that $M_{\tilde{a}}^{P}, M_{\tilde{a}}^{N}$ and $M_{\tilde{a}}^{C}$ satisfy Definition 2.1(M.i) - (M.iv) since $\tilde{a}$ is continuous and has a compact support. Since $M_{\tilde{a}}^{P}\left(\tilde{a}_{\alpha}\right)=1$ and $M_{\tilde{a}}^{N}\left(\tilde{a}_{\alpha}\right)=1-\alpha$ and $M_{\tilde{a}}^{C}\left(\tilde{a}_{\alpha}\right)=1-\alpha / 2$ from $(2.6)-(2.8)$, the corresponding mean values $\tilde{E}(\tilde{a})$ are reduced to

$$
\begin{aligned}
\tilde{E}^{P}(\tilde{a}) & :=\int_{0}^{1} g\left(\tilde{a}_{\alpha}\right) \mathrm{d} \alpha \\
\tilde{E}^{N}(\tilde{a}) & :=\int_{0}^{1} 2(1-\alpha) g\left(\tilde{a}_{\alpha}\right) \mathrm{d} \alpha \\
\tilde{E}^{C}(\tilde{a}) & :=\int_{0}^{1} \frac{4}{3}\left(1-\frac{\alpha}{2}\right) g\left(\tilde{a}_{\alpha}\right) \mathrm{d} \alpha .
\end{aligned}
$$


They are called a 'possibility mean', a 'necessity mean' and a 'credibility mean' of the fuzzy number $\tilde{a}$ respectively.

In the same idea, we introduce mean values of fuzzy random variables $\tilde{X}$ as follows.

$$
\begin{aligned}
& E(\tilde{E}(\tilde{X})(\cdot)) \\
:= & E\left(\int_{0}^{1} M_{\tilde{X}(\cdot)}\left(\tilde{X}_{\alpha}(\cdot)\right) g\left(\tilde{X}_{\alpha}(\cdot)\right) \mathrm{d} \alpha / \int_{0}^{1} M_{\tilde{X}(\cdot)}\left(\tilde{X}_{\alpha}(\cdot)\right) \mathrm{d} \alpha\right),
\end{aligned}
$$

where $\tilde{X}_{\alpha}(\cdot)=\left[\tilde{X}_{\alpha}^{-}(\cdot), \tilde{X}_{\alpha}^{+}(\cdot)\right]$.

\section{AMERICAN PUT OPTION WITH UNCERTAINTY OF STOCK PRICES}

In this section, we formulate American put option with uncertainty of stock prices by fuzzy random variables. Let $\mathbb{T}:=\{0,1,2, \cdots, T\}$ be the time space with an expiration date $T$ similarly to the previous section, and take a probability space $\Omega:=\mathbb{R}^{T+1}$. Let $r(r>0)$ be an interest rate of a bond price, which is a riskless asset, and put a discount rate $\beta=1 /(1+r)$. Define a 'stock price process' $\left\{S_{t}\right\}_{t=0}^{T}$ as follows: An initial stock price $S_{0}$ is a positive constant and stock prices are given by

$$
S_{t}:=S_{0} \prod_{s=1}^{t}\left(1+Y_{s}\right) \quad \text { for } t=1,2, \cdots, T,
$$

where $\left\{Y_{t}\right\}_{t=1}^{T}$ is a uniform integrable sequence of independent, identically distributed real random variables on $[-1, \infty)$ such that $E\left(Y_{t}\right)=r$ for all $t=1,2, \cdots, T$. The $\sigma$-fields $\left\{\mathcal{M}_{t}\right\}_{t=0}^{T}$ are defined as follows: $\mathcal{M}_{0}$ is the completion of $\{\emptyset, \Omega\}$ and $\mathcal{M}_{t}(t=1,2, \cdots, T)$ denote the complete $\sigma$-fields generated by $\left\{Y_{1}, Y_{2} \cdots Y_{t}\right\}$.

We consider a finance model where the stock price process $\left\{S_{t}\right\}_{t=0}^{T}$ takes fuzzy values. Now we give fuzzy values by triangular fuzzy numbers for simplicity. Let $\left\{a_{t}\right\}_{t=0}^{T}$ be an $\mathcal{M}_{t}$-adapted stochastic process such that $0<a_{t}(\omega) \leq S_{t}(\omega)$ for $\omega \in \Omega$. A 'stock price process with fuzzy values' are represented by a sequence of fuzzy random variables $\left\{\tilde{S}_{t}\right\}_{t=0}^{T}$ :

$$
\tilde{S}_{t}(\omega)(x):=L\left(\left(x-S_{t}(\omega)\right) / a_{t}(\omega)\right)
$$

for $t \in \mathbb{T}, \omega \in \Omega$ and $x \in \mathbb{R}$, where $L(x):=\max \{1-|x|, 0\}(x \in \mathbb{R})$ is the triangle shape function (Fig.3.1). Hence, $a_{t}(\omega)$ is a spread of triangular fuzzy numbers $\tilde{S}_{t}(\omega)$ and corresponds to the amount of fuzziness in the process. Then, $a_{t}(\omega)$ should be an increasing function of the stock price $S_{t}(\omega)$ (see Assumption $\mathrm{S}$ in the next section). 


\section{Figure 3.1}

Fig. 3.1. Fuzzy random variable $\tilde{S}_{t}(\omega)(x)$.

Let $K(K>0)$ be a 'strike price'. The 'price process' $\left\{\tilde{P}_{t}\right\}_{t=0}^{T}$ of American put option under uncertainty is represented by

$$
\tilde{P}_{t}(\omega):=\beta^{t}\left(1_{\{K\}}-\tilde{S}_{t}(\omega)\right) \vee 1_{\{0\}} \text { for } t=0,1,2, \cdots, T,
$$

where $\vee$ is given by $(2.1)$, and $1_{\{K\}}$ and $1_{\{0\}}$ denote the crisp number $K$ and zero respectively. An 'exercise time' in American put option is given by a stopping time $\tau$ with values in $\mathbb{T}$. For an exercise time $\tau$, we define

$$
\tilde{P}_{\tau}(\omega):=\tilde{P}_{t}(\omega) \quad \text { if } \tau(\omega)=t \quad \text { for } t=0,1,2, \cdots, T \text {, and } \omega \in \Omega .
$$

Then, from Lemma 2.1, $\tilde{P}_{\tau}$ is a fuzzy random variable. Now we analyze (3.3) by $\alpha$-cuts technique of fuzzy numbers. The $\alpha$-cuts of fuzzy random variables (3.2) are

$$
\tilde{S}_{t, \alpha}(\omega)=\left[S_{t}(\omega)-(1-\alpha) a_{t}(\omega), S_{t}(\omega)+(1-\alpha) a_{t}(\omega)\right], \quad \omega \in \Omega,
$$

and so we put

$$
\tilde{S}_{t, \alpha}^{ \pm}(\omega):=S_{t}(\omega) \pm(1-\alpha) a_{t}(\omega), \quad \omega \in \Omega
$$

for $t \in \mathbb{T}$ and $\alpha \in[0,1]$. Therefore, the $\alpha$-cuts of (3.3) are

$$
\begin{aligned}
& \tilde{P}_{t, \alpha}(\omega) \\
= & {\left[\tilde{P}_{t, \alpha}^{-}(\omega), \tilde{P}_{t, \alpha}^{+}(\omega)\right] } \\
:= & {\left[\beta^{t} \max \left\{K-\tilde{S}_{t, \alpha}^{+}(\omega), 0\right\}, \beta^{t} \max \left\{K-\tilde{S}_{t, \alpha}^{-}(\omega), 0\right\}\right], }
\end{aligned}
$$

and we obtain $E\left(\max _{t \in \mathbb{T}} \sup _{\alpha \in[0,1]} \tilde{P}_{t, \alpha}^{+}\right) \leq K<\infty$ since $\tilde{S}_{t, \alpha}^{-}(\omega) \geq 0$, where $E(\cdot)$ is the expectation with respect to some risk-neutral equivalent martingale measure([3]). Let $\omega \in \Omega$ and let $\tau$ be an exercise time. From $(2.5)$, the evaluation of the fuzzy number $\tilde{P}_{\tau}(\omega)$ is given by

$$
\begin{aligned}
& \tilde{E}\left(\tilde{P}_{\tau}\right)(\omega):=\tilde{E}\left(\tilde{P}_{\tau}(\omega)\right) \\
= & \int_{0}^{1} M_{\tilde{P}_{\tau}(\omega)}\left(\tilde{P}_{\tau, \alpha}(\omega)\right) g\left(\tilde{P}_{\tau, \alpha}(\omega)\right) \mathrm{d} \alpha / \int_{0}^{1} M_{\tilde{P}_{\tau}(\omega)}\left(\tilde{P}_{\tau, \alpha}(\omega)\right) \mathrm{d} \alpha, \omega \in \Omega .
\end{aligned}
$$


From (2.12), we put the expectation of (3.8) by $\boldsymbol{P}(\tau)$ :

$$
\begin{aligned}
& \boldsymbol{P}(\tau):=E\left(\tilde{E}\left(\tilde{P}_{\tau}\right)(\cdot)\right) \\
= & E\left(\int_{0}^{1} M_{\tilde{P}_{\tau}(\cdot)}\left(\tilde{P}_{\tau, \alpha}(\cdot)\right) g\left(\tilde{P}_{\tau, \alpha}(\cdot)\right) \mathrm{d} \alpha / \int_{0}^{1} M_{\tilde{P}_{\tau}(\cdot)}\left(\tilde{P}_{\tau, \alpha}(\cdot)\right) \mathrm{d} \alpha\right) .
\end{aligned}
$$

Then $\boldsymbol{P}(\tau)$ means an evaluation of the expected price of American put option when $\tau$ is an exercise time. In American put option, we must maximize the expected values $\boldsymbol{P}(\tau)$ of the price process by stopping times $\tau$. Put the 'optimal expected price' by

$$
\boldsymbol{V}:=\sup _{\tau: \tau \leq T} \boldsymbol{P}(\tau) .
$$

In the next section, this paper discusses the following optimal stopping problem regarding American put option with fuzziness.

Problem P. Find a stopping time $\tau^{*}\left(\tau^{*} \leq T\right)$ and the optimal expected price $\boldsymbol{V}$ such that

$$
\boldsymbol{P}\left(\tau^{*}\right)=\boldsymbol{V}
$$

Then, $\tau^{*}$ is called an 'optimal exercise time'. On the other hand, for an exercise time $\tau$, the expectation of the fuzzy random variable $\tilde{P}_{\tau}$ is a fuzzy number whose $\alpha$-cut is a closed interval

$$
E\left(\tilde{P}_{\tau}\right)_{\alpha}=E\left(\tilde{P}_{\tau, \alpha}\right)=\left[E\left(\tilde{P}_{\tau, \alpha}^{-}\right), E\left(\tilde{P}_{\tau, \alpha}^{+}\right)\right] \quad \text { for } \alpha \in[0,1],
$$

where $\tilde{P}_{\tau(\omega), \alpha}(\omega)=\left[\tilde{P}_{\tau(\omega), \alpha}^{-}(\omega), \tilde{P}_{\tau(\omega), \alpha}^{+}(\omega)\right]$ is the $\alpha$-cut of fuzzy number $\tilde{P}_{\tau}(\omega)$. The expectation of the fuzzy random variable $\tilde{P}_{\tau}$ is a fuzzy number(see $(2.2))$

$$
E\left(\tilde{P}_{\tau}\right)(x):=\sup _{\alpha \in[0,1]} \min \left\{\alpha, 1_{E\left(\tilde{P}_{\tau}\right)_{\alpha}}(x)\right\}, \quad x \in \mathbb{R},
$$

where $E\left(\tilde{P}_{\tau}\right)_{\alpha}=\left[\int_{\Omega} \tilde{P}_{\tau, \alpha}^{-}(\omega) \mathrm{d} P(\omega), \int_{\Omega} \tilde{P}_{\tau, \alpha}^{+}(\omega) \mathrm{d} P(\omega)\right]$. Thus, by (2.5) we have another estimation regarding the price process $\left\{\tilde{P}_{t}\right\}_{t \in \mathbb{T}}$ of American put option at time $\tau$ :

$$
\begin{aligned}
& \tilde{E}\left(E\left(\tilde{P}_{\tau}\right)\right) \\
= & \int_{0}^{1} M_{E\left(\tilde{P}_{\tau}\right)}\left(E\left(\tilde{P}_{\tau}\right)_{\alpha}\right) g\left(E\left(\tilde{P}_{\tau}\right)_{\alpha}\right) \mathrm{d} \alpha / \int_{0}^{1} M_{E\left(\tilde{P}_{\tau}\right)}\left(E\left(\tilde{P}_{\tau}\right)_{\alpha}\right) \mathrm{d} \alpha .
\end{aligned}
$$

However, (3.14) coincides with (3.9) in cases of the possibility mean, the necessity mean and the credibility mean in Example 2.1. Now we discuss it in the following more gerneral framework. 
Assumption M. There exists a nonincreasing function $\rho:[0,1] \mapsto$ $[0,1]$ such that

$$
M_{\tilde{a}}\left(\tilde{a}_{\alpha}\right)=\rho(\alpha), \quad \alpha \in[0,1] \quad \text { for all } \tilde{a} \in \mathcal{R} .
$$

In Example 2.1, the possibility evaluation measure $M_{\tilde{a}}^{P}$, the necessity evaluation measure $M_{\tilde{a}}^{N}$ and the credibility evaluation measure $M_{\tilde{a}}^{C}$ have the following nonincreasing functions $\rho$ in Assumption M:

$\rho^{P}(\alpha):=1 \quad:$ in case of the possibility evaluation measure;

$\rho^{N}(\alpha):=1-\alpha \quad:$ in case of the necessity evaluation measure;

$\rho^{C}(\alpha):=1-\alpha / 2$ : in case of the credibility evaluation measure.

Lemma 3.1. Suppose Assumption M holds. Let $\tau$ be an exercise time. Then it holds that

$$
\begin{aligned}
\boldsymbol{P}(\tau) & =E\left(\int_{0}^{1} M_{\tilde{P}_{\tau}(\cdot)}\left(\tilde{P}_{\tau, \alpha}(\cdot)\right) g\left(\tilde{P}_{\tau, \alpha}(\cdot)\right) \mathrm{d} \alpha / \int_{0}^{1} M_{\tilde{P}_{\tau}(\cdot)}\left(\tilde{P}_{\tau, \alpha}(\cdot)\right) \mathrm{d} \alpha\right) \\
& =\int_{0}^{1} M_{E\left(\tilde{P}_{\tau}\right)}\left(E\left(\tilde{P}_{\tau}\right)_{\alpha}\right) E\left(g\left(\tilde{P}_{\tau, \alpha}\right)\right) \mathrm{d} \alpha / \int_{0}^{1} M_{E\left(\tilde{P}_{\tau}\right)}\left(E\left(\tilde{P}_{\tau}\right)_{\alpha}\right) \mathrm{d} \alpha \\
& =\int_{0}^{1} M_{E\left(\tilde{P}_{\tau}\right)}\left(E\left(\tilde{P}_{\tau}\right)_{\alpha}\right) g\left(E\left(\tilde{P}_{\tau}\right)_{\alpha}\right) \mathrm{d} \alpha / \int_{0}^{1} M_{E\left(\tilde{P}_{\tau}\right)}\left(E\left(\tilde{P}_{\tau}\right)_{\alpha}\right) \mathrm{d} \alpha \\
& =\tilde{E}\left(E\left(\tilde{P}_{\tau}\right)\right)
\end{aligned}
$$

Proof. From (2.2) and the definition (2.3) of $g$, we have

$$
g\left(E\left(\tilde{P}_{\tau}\right)_{\alpha}\right)=g\left(E\left(\tilde{P}_{\tau, \alpha}\right)\right)=E\left(g\left(\tilde{P}_{\tau, \alpha}\right)\right) .
$$

So, the last two equalities hold in (3.15). Under Assumption M, we have $M_{E\left(\tilde{P}_{\tau}\right)}\left(E\left(\tilde{P}_{\tau}\right)_{\alpha}\right)=M_{\tilde{P}_{\tau}}\left(\tilde{P}_{\tau, \alpha}(\cdot)\right)=\rho(\alpha)$. By Fubini's theorem, we obtain

$$
\begin{aligned}
& \int_{0}^{1} M_{E\left(\tilde{P}_{\tau}\right)}\left(E\left(\tilde{P}_{\tau}\right)_{\alpha}\right) E\left(g\left(\tilde{P}_{\tau, \alpha}\right)\right) \mathrm{d} \alpha / \int_{0}^{1} M_{E\left(\tilde{P}_{\tau}\right)}\left(E\left(\tilde{P}_{\tau}\right)_{\alpha}\right) \mathrm{d} \alpha \\
= & \int_{0}^{1} \rho(\alpha) E\left(g\left(\tilde{P}_{\tau, \alpha}\right)\right) \mathrm{d} \alpha / \int_{0}^{1} \rho(\alpha) \mathrm{d} \alpha \\
= & E\left(\int_{0}^{1} \rho(\alpha) g\left(\tilde{P}_{\tau, \alpha}(\cdot)\right) \mathrm{d} \alpha / \int_{0}^{1} \rho(\alpha) \mathrm{d} \alpha\right) \\
= & \boldsymbol{P}(\tau) .
\end{aligned}
$$

These complete the proof of this lemma. 
4. The optimal EXPECTED PRICE AND THE OPTIMAL EXERCise TIME

In this section, we discuss the optimal fuzzy price $\boldsymbol{V}$ and the optimal exercise time $\tau^{*}$, by using dynamic programming approach. Now we introduce an assumption.

Assumption S. The stochastic process $\left\{a_{t}\right\}_{t=0}^{T}$ is represented by

$$
a_{t}(\omega):=c S_{t}(\omega), \quad t=0,1,2, \cdots, T, \omega \in \Omega,
$$

where $c$ is a constant satisfying $0<c<1$.

\section{Figure 4.1}

Fig. 4.1. Fuzzy stock price $\tilde{S}_{t}(\omega)(x)$ and Assumption S.

Assumption $\mathrm{S}$ is reasonable since $a_{t}(\omega)$ means a size of fuzziness and it should depend on the volatility and the stock price $S_{t}(\omega)$ because one of the most difficulties is estimation of the actual volatility ([11, Sect.7.5.1], Fig.4.1). In this model, we represent by $c$ the fuzziness of the volatility, and we call $c$ a 'fuzzy factor' of the process. From now on, we suppose that Assumption $\mathrm{S}$ and Assumption M hold. By putting $b^{ \pm}(\alpha):=1 \pm(1-\alpha) c(\alpha \in[0,1])$, from (3.2) we have

$$
\begin{aligned}
\tilde{S}_{t, \alpha}^{ \pm}(\omega) & =S_{t}(\omega) \pm(1-\alpha) a_{t}(\omega) \\
& =b^{ \pm}(\alpha) S_{t}(\omega) \\
& =b^{ \pm}(\alpha) S_{0} \prod_{s=1}^{t}\left(1+Y_{s}(\omega)\right), \quad \omega \in \Omega
\end{aligned}
$$

for $t \in \mathbb{T}$ and $\alpha \in[0,1]$. Then, from (3.7) and (4.1), we have the fuzzy price process:

$$
\tilde{P}_{\tau, \alpha}^{ \pm}(\omega)=\beta^{\tau(\omega)} \max \left\{K-b^{\mp}(\alpha) S_{\tau}(\omega), 0\right\}, \quad \omega \in \Omega .
$$

For an exercise time $\tau$, we define a random variable

$$
\begin{aligned}
& \Pi_{\tau}(\omega):=\tilde{E}\left(\tilde{P}_{\tau}\right)(\omega) \\
= & \int_{0}^{1} M_{\tilde{P}_{\tau}(\omega)}\left(\tilde{P}_{\tau, \alpha}(\omega)\right) g\left(\tilde{P}_{\tau, \alpha}(\omega)\right) \mathrm{d} \alpha / \int_{0}^{1} M_{\tilde{P}_{\tau}(\omega)}\left(\tilde{P}_{\tau, \alpha}(\omega)\right) \mathrm{d} \alpha
\end{aligned}
$$


$\omega \in \Omega$. From Lemma 3.1, $\boldsymbol{P}(\tau)=E\left(\Pi_{\tau}\right)$ is the evaluated price of American put option when $\tau$ is an exercise time. Now we give an optimal exercise time for Problem $\mathrm{P}$ and we discuss an iterative method to obtain the optimal expected price $\boldsymbol{V}$ in (3.14). To analyze the optimal fuzzy price $\boldsymbol{V}$, we put

$$
\boldsymbol{V}_{t}(y)=\sup _{\tau: \text { stopping times, } t \leq \tau \leq T} E\left(\beta^{-t} \Pi_{\tau} \mid S_{t}=y\right)
$$

for $t=0,1,2, \cdots, T$ and an initial stock price $y(y>0)$. Then we note that $\boldsymbol{V}=\boldsymbol{V}_{0}(y)$. Next, we define the following stochastic sequence $\left\{Z_{t}\right\}_{t=0}^{T}$ : Let $t \in \mathbb{T}$. Define

$$
Z_{t}:=\underset{\tau: \text { stopping times, } t \leq \tau \leq T}{\operatorname{ess} \sup _{\tau}} E\left(\Pi_{\tau} \mid \mathcal{M}_{t}\right) .
$$

Refer to [8, Prop.4-1-1] regarding the essential supremum. The random variables $Z_{t}$ is called 'Snell's envelope'([8]). Hence we obtain the following recursive equation for the stochastic sequence $\left\{Z_{t}\right\}_{t=0}^{T}$ and the optimal fuzzy price $\boldsymbol{V}$.

Lemma 4.1 (Recurrsive equation). The following (i) and (ii) hold.

(i) $\boldsymbol{V}=E\left(Z_{0} \mid S_{0}=y\right)$.

(ii) $Z_{t}(\omega)=\max \left\{\Pi_{t}(\omega), E\left(Z_{t+1} \mid \mathcal{M}_{t}\right)(\omega)\right\}$, a.a. $\omega, t=0,1, \cdots, T-1$.

Proof. (i) is trivial from the definition of the essential supremum and (3.10). Next we prove (ii). Let $t=0,1, \cdots, T-1$. From the definition of the conditional expectation and the monotone convergence theorem, we have

$$
\begin{aligned}
& \max \left\{\Pi_{t}, E\left(Z_{t+1} \mid \mathcal{M}_{t}\right)\right\} \\
= & \max \left\{\Pi_{t}, E\left(\operatorname{ess}_{\tau: t+1 \leq \tau \leq T} E\left(\Pi_{\tau} \mid \mathcal{M}_{t+1}\right) \mid \mathcal{M}_{t}\right)\right\} \\
= & \max \left\{\Pi_{t}, \operatorname{ess~sup}_{\tau: t+1 \leq \tau \leq T} E\left(\Pi_{\tau} \mid \mathcal{M}_{t}\right)\right\} \\
= & \operatorname{ess}_{\tau: t \leq \tau \leq T} E\left(\Pi_{\tau} \mid \mathcal{M}_{t}\right) \\
= & Z_{t} \text { almost surely. }
\end{aligned}
$$

Thus we obtain (ii), and therefore the proof of this lemma is completed.

Hence we have the following representation about (4.3). 
Lemma 4.2. Let $\tau$ be a stopping time satisfying $\tau \leq T$. Let (4.3) be the possibility mean in Example 2.1. Then there exists a function $f$ on $(0, \infty)$ such that

$$
\Pi_{\tau}(\omega)=\beta^{\tau(\omega)} f\left(S_{\tau}(\omega)\right), \quad \omega \in \Omega .
$$

The representation (4.6) also holds in case of the necessity mean and the credibility mean. Here, the function $f$ is given by $f=f^{P}\left(f=f^{N}, f=\right.$ $f^{C}$ ) corresponding to the possibility mean (the necessity mean, the credibility mean respectively), where

$$
\begin{aligned}
& f^{P}(y):= \begin{cases}K-y-\frac{1}{2} c y(2 \lambda-1)+\lambda \varphi^{P, 1}(y) & \text { if } 0<y<K \\
(1-\lambda) \varphi^{P, 2}(y) & \text { if } y \geq K,\end{cases} \\
& f^{N}(y):= \begin{cases}K-y-\frac{2}{3} c y(2 \lambda-1)+\lambda \varphi^{N, 1}(y) & \text { if } 0<y<K \\
(1-\lambda) \varphi^{N, 2}(y) & \text { if } y \geq K,\end{cases} \\
& f^{C}(y):= \begin{cases}K-y-\frac{5}{9} c y(2 \lambda-1)+\lambda \varphi^{C, 1}(y) & \text { if } 0<y<K \\
(1-\lambda) \varphi^{C, 2}(y) & \text { if } y \geq K,\end{cases}
\end{aligned}
$$

with functions $\varphi^{P, 1}, \varphi^{P, 2}, \varphi^{N, 1}, \varphi^{N, 2}, \varphi^{C, 1}, \varphi^{C, 2}$ on $(0, \infty)$ : For $y>0$,

$$
\begin{aligned}
\varphi^{P, 1}(y) & :=\frac{1}{2 c y} \max \left\{0, K_{2}(y)\right\}^{2}, \\
\varphi^{P, 2}(y) & :=\frac{1}{2 c y} \max \left\{0, K_{1}(y)\right\}^{2}, \\
\varphi^{N, 1}(y) & :=\frac{1}{c y} \max \left\{0, K_{2}(y)\right\}^{2}-\frac{1}{3(c y)^{2}} \max \left\{0, K_{2}(y)\right\}^{3}, \\
\varphi^{N, 2}(y) & :=\frac{1}{c y} \max \left\{0, K_{1}(y)\right\}^{2}-\frac{1}{3(c y)^{2}} \max \left\{0, K_{1}(y)\right\}^{3}, \\
\varphi^{C, 1}(y) & :=\frac{2}{3 c y} \max \left\{0, K_{2}(y)\right\}^{2}-\frac{1}{9(c y)^{2}} \max \left\{0, K_{2}(y)\right\}^{3}, \\
\varphi^{C, 2}(y) & :=\frac{2}{3 c y} \max \left\{0, K_{2}(y)\right\}^{2}-\frac{1}{9(c y)^{2}} \max \left\{0, K_{2}(y)\right\}^{3} .
\end{aligned}
$$

Here we put $K_{1}(y):=K-y+c y$ and $K_{2}(y):=-K+y+c y$.

Proof. Fix any $\omega \in \Omega$. From (2.3) and (4.2), we have

$$
\begin{aligned}
& g\left(\tilde{P}_{\tau, \alpha}(\omega)\right)=\lambda \tilde{P}_{\tau, \alpha}^{-}(\omega)+(1-\lambda) \tilde{P}_{\tau, \alpha}^{+}(\omega) \\
=\lambda \beta^{\tau(\omega)} \max \left\{K-b^{+}(\alpha) S_{\tau}(\omega), 0\right\} & \\
& +(1-\lambda) \beta^{\tau(\omega)} \max \left\{K-b^{-}(\alpha) S_{\tau}(\omega), 0\right\} .
\end{aligned}
$$


It is represented as $g\left(\tilde{P}_{\tau, \alpha}(\omega)\right)=\beta^{\tau(\omega)} h\left(S_{\tau}(\omega), \alpha\right)$ with a function $h$ on $(0, \infty) \times[0,1]$ such that

$$
h(y, \alpha):= \begin{cases}K-b^{\lambda}(\alpha) y & \text { if } 0<y<K / b^{+}(\alpha) \\ (1-\lambda)\left(K-b^{-}(\alpha) y\right) & \text { if } K / b^{+}(\alpha) \leq y<K / b^{-}(\alpha) \\ 0 & \text { if } y \geq K / b^{-}(\alpha)\end{cases}
$$

where $b^{\lambda}(\alpha):=\lambda b^{+}(\alpha)+(1-\lambda) b^{-}(\alpha)=1+(2 \lambda-1)(1-\alpha) c$. Then, in case of necessity mean values, we can easily calculate that the integration of $2(1-\alpha) h(y, \alpha)$ is given by the following function $f^{N}$ on $(0, \infty)$ :

$$
\begin{aligned}
f^{N}(y) & :=\int_{0}^{1} 2(1-\alpha) h(y, \alpha) \mathrm{d} \alpha \\
& = \begin{cases}K-y-\frac{2}{3} c y(2 \lambda-1)+\lambda \varphi^{N, 1}(y) & \text { if } 0<y<K \\
(1-\lambda) \varphi^{N, 2}(y) & \text { if } y \geq K,\end{cases}
\end{aligned}
$$

where $\varphi^{N, 1}$ and $\varphi^{N, 2}$ are given in the statements of this lemma. Thus we have

$$
\begin{aligned}
& \Pi_{\tau}(\omega)=\int_{0}^{1} 2(1-\alpha) g\left(\tilde{P}_{\tau, \alpha}(\omega)\right) \mathrm{d} \alpha \\
= & \int_{0}^{1} \beta^{\tau(\omega)} 2(1-\alpha) h\left(S_{\tau}(\omega), \alpha\right) \mathrm{d} \alpha=\beta^{\tau(\omega)} f^{N}\left(S_{\tau}(\omega)\right) .
\end{aligned}
$$

We can easily check the other cases in a similar way. Thus we obtain this lemma.

\section{Figure 4.2}

Fig. 4.2. The function $f^{P}(y)(\lambda=1 / 2, c=0.05, K=35)$.

Fig.4.2 indicates the function $f^{P}(y)$, whch is given by (4.7), in case of the possibility measure at $\lambda=1 / 2, c=0.05, K=35$. Since the stock price process $\left\{S_{t}\right\}_{t=0}^{T}$ is Markov by (3.1), we obtain the following results from Lemma 4.2 .

Theorem 4.1 (Optimality equation). 
(i) The optimal expected price $\boldsymbol{V}=\boldsymbol{V}_{0}(y)$ with an initial stock price $y(y>0)$ is given by the following backward recursive equations (4.11) and (4.12): For $t=0,1, \cdots, T-1, y>0$,

$$
\begin{gathered}
\boldsymbol{V}_{t}(y)=\max \left\{\beta E\left(\boldsymbol{V}_{t+1}\left(y\left(1+Y_{1}\right)\right)\right), f(y)\right\}, \\
\boldsymbol{V}_{T}(y)=f(y) .
\end{gathered}
$$

(ii) Define a stopping time

$$
\tau^{*}(\omega):=\inf \left\{t \in \mathbb{T} \mid V_{t}\left(S_{t}(\omega)\right)=f\left(S_{t}(\omega)\right)\right\}, \quad \omega \in \Omega,
$$

where the infimum of the empty set is understood to be T. Then, $\tau^{*}$ is an optimal exercise time for Problem $P$, and the optimal value of American put option is

$$
\boldsymbol{V}=\boldsymbol{V}_{0}(y)=\boldsymbol{P}\left(\tau^{*}\right)
$$

for an initial stock price $y>0$.

Proof. (i) Taking expectations of Lemma 4.1(ii) with $E\left(\cdot \mid S_{t}=y\right.$ ), from (3.1), (4.3) and (4.6) we have

$$
\begin{aligned}
\beta^{t} \boldsymbol{V}_{t}(y) & =\max \left\{\beta^{t} f(y), \beta^{t+1} E\left(\boldsymbol{V}_{t+1}\left(y S_{1}\right)\right)\right\} \\
& =\max \left\{\beta^{t} f(y), \beta^{t+1} E\left(\boldsymbol{V}_{t+1}\left(y\left(1+Y_{1}\right)\right)\right)\right\} .
\end{aligned}
$$

While by (4.4) and (4.6), we also have

$$
\boldsymbol{V}_{T}(y)=E\left(\beta^{T} \Pi_{T} \mid S_{T}=y\right)=E\left(f\left(S_{T}\right) \mid S_{T}=y\right)=f(y) .
$$

Thus we obtain (4.11) and (4.12) from these equations.

(ii) Using the supermartingale $\left\{Z_{t}\right\}_{t=0}^{T}$, from Lemma 4.1(i) and [8, Prop.6-1-3] we have

$$
\boldsymbol{V}=E\left(Z_{0} \mid S_{0}=y\right)=E\left(\Pi_{\tau^{*}} \mid S_{0}=y\right)=\boldsymbol{P}\left(\tau^{*}\right) .
$$

Therefore, $\tau^{*}$ is an optimal exercise time for Problem $\mathrm{P}$ and we also obtain (4.14).

\section{A NUMERICAL EXAMPLE}

Now we give a numerical example to illustrate our idea in Sections 3 and 4 .

Example 5.1. We consider CRR type American put option model (see Ross [11, Sect.7.4]). Put an expiration date $T=10$, an interest 
rate of a bond $r=0.05$, a fuzzy factor $c=0.05$, an initial stock price $y=30$ and a strike price $K=35$. Assume that $\left\{Y_{t}\right\}_{t=1}^{T}$ is a uniform sequence of independent, identically distributed real random variables such that

$$
Y_{t}:= \begin{cases}e^{\sigma}-1 & \text { with probability } p \\ e^{-\sigma}-1 & \text { with probability }(1-p)\end{cases}
$$

for all $t=1,2, \cdots, T$, where $\sigma=0.25$ is a volatility of the stock and the probability $p$ is given by $p=\left(1+r-e^{-\sigma}\right) /\left(e^{\sigma}-e^{-\sigma}\right)$. Then we have the risk neutral condition $E\left(Y_{t}\right)=r$. This model has a binomial form where the stock will go up with probability $p$ and will go down with probability $1-p$. If we take a $\lambda$-weighting function $g$ with a pessimisticoptimistic index $\lambda=1 / 2$ in (2.3), Fig.5.1 shows the corresponding optimal expected price of American put option $\boldsymbol{V}_{0}(y)$ for each initial stock price $y$ in case of the possibility mean, and then the corresponding optimal exercise time is reduced to

$$
\begin{aligned}
\tau^{*}(\omega) & =\inf \left\{t \in \mathbb{T} \mid \boldsymbol{V}_{t}\left(S_{t}(\omega)\right)=f^{P}\left(S_{t}(\omega)\right)\right\} \\
& =\inf \left\{t \in \mathbb{T} \mid S_{t}(\omega) \leq y_{t}^{*}\right\},
\end{aligned}
$$

where $y_{t}^{*}$ is the stopping boundary price for the stock prices $S_{t}$ which is given by $\left[0, y_{t}^{*}\right]=\left\{y \in[0, \infty) \mid \boldsymbol{V}_{t}(y)=f^{P}(y)\right\}$ and we can easily calculate it (see Table 5.1).

Table 5.1. The stopping boundary price $y_{t}^{*}$

$$
(\lambda=1 / 2 ; c=0.05 ; K=35 ; y=30)
$$

\begin{tabular}{c|cccccc}
\hline$t$ & 0 & 1 & 2 & 3 & 4 & 5 \\
\hline$y_{t}^{*}$ & 24.895 & 24.976 & 25.131 & 25.277 & 25.484 & 25.798 \\
\hline & & 6 & 7 & 8 & 9 & 10 \\
\cline { 2 - 6 } & & 26.052 & 26.921 & 27.101 & 29.807 & 30.000 \\
\hline
\end{tabular}

\section{Figure 5.1}


Fig. 5.1. The optimal expected price of American put option $\boldsymbol{V}_{0}(y)$ and the function $f^{P}(y)(\lambda=1 / 2, c=0.05, K=35, y$ : initial stock price)

The optimal expected price of American put option $\boldsymbol{V}_{0}(y)$ changes corresponding to the pessimistic-optimistic index $\lambda(0 \leq \lambda \leq 1)$ in the $\lambda$-weighting function (2.3), where $\lambda$ means the pessimistic degree in the writer's decision making. By the optimality equation in Theorem 4.1(i), we can calculate the optimal expected price $\boldsymbol{V}_{0}(y)$. The initial option price is $K-y=5$, but Table 5.2 shows the optimal expected price of American put option $\boldsymbol{V}_{0}(y)$ at initial stock price $y=30$.

Table 5.2. The optimal expected price $\boldsymbol{V}_{0}(y)$

$(\lambda=1 / 3,1 / 2,2 / 3 ; c=0.05 ; K=35 ; y=30)$

\begin{tabular}{lc|ccccc}
\hline & $\lambda=0$ & $\lambda=1 / 3$ & $\lambda=1 / 2$ & $\lambda=2 / 3$ & $\lambda=1$ \\
\hline \hline The possibility mean $\tilde{E}^{P}$ & 7.780 & 7.482 & 7.396 & 7.311 & 7.013 \\
\hline The necessity mean & $\tilde{E}^{N}$ & 7.908 & 7.567 & 7.396 & 7.226 & 6.885 \\
\hline The credibility mean $\tilde{E}^{C}$ & 7.822 & 7.538 & 7.396 & 7.255 & 6.971 \\
\hline
\end{tabular}

We can utilize the results of $\lambda=1$ and $\lambda=0$ as confidence intervals in fuzzy and stochastic environment. For example, from the possibility mean case of Table 5.2, the interval [7.013, 7.780] can be taken as a kind of confidence intervals for the optimal expected price $\boldsymbol{V}_{0}(y)$. Similarly in the necessity mean case (the credibility mean case resp.), we can give the corresponding confidence intervals by $[6.885,7.908]([6.971,7.822])$.

\section{Acknowledgments.}

The author would like to thank the referees for valuable comments and suggestions. Especially they show us several references.

\section{References}

1. C.Carlsson and R.Fullér, On possibilistic mean value and variance of fuzzy numbers, Fuzzy Sets and Systems 122 (2001) 315-326.

2. C.Carlsson and R.Fullér, A fuzzy approach to real option valuation, Fuzzy Sets and Systems 139 (2003) 297-312. 
3. R.J.Elliott and P.E.Kopp Mathematics of Financial Markets (Springer, New York, 1999).

4. P.Fortemps and M.Roubens, Ranking and defuzzification methods based on area compensation, Fuzzy Sets and Systems 82 (1996) 319-330.

5. R.Goetshel and W.Voxman, Elementary fuzzy calculus, Fuzzy Sets and Systems 18 (1986) 31-43.

6. H.Kwakernaak, Fuzzy random variables - I. Definitions and theorem, Inform. Sci. 15 (1978) 1-29.

7. H.Kwakernaak, Fuzzy random variables - II. Algorithms and examples for the discrete case, Inform. Sci. 17 (1979) 253-278.

8. J.Neveu, Discrete-Parameter Martingales (North-Holland, New York, 1975).

9. S.R.Pliska Introduction to Mathematical Finance: Discrete-Time Models (Blackwell Publ., New York, 1997).

10. M.L.Puri and D.A.Ralescu, Fuzzy random variables, J. Math. Anal. Appl. 114 (1986) 409-422.

11. S.M.Ross, An Introduction to Mathematical Finance (Cambridge Univ. Press, Cambridge , 1999).

12. A.N.Shiryayev, Optimal Stopping Rules (Springer, New York, 1979).

13. M.R.Simonelli, Fuzziness in valuing financial instruments by certainty equivalents, European J. Oper. Res. 135 (2001) 296-302.

14. Z.Wang and G.J.Klir, Fuzzy Measure Theory (Plenum Press, New York, 1993).

15. G.Wang and Y.Zhang, The theory of fuzzy stochastic processes, Fuzzy Sets and Systems 51 (1992) 161-178.

16. Y.Yoshida, M.Yasuda, J.Nakagami and M.Kurano, Optimal stopping problems in a stochastic and fuzzy system, J. Math. Analy. and Appl. 246 (2000) 135-149.

17. Y.Yoshida, M.Yasuda, J.Nakagami and M.Kurano, Decision making for American options with uncertainty of stock prices in financial engineering, Proceedings of IPMU 2002, (the 9th International Conference on Information Processing and Management of Uncertainty in Knowledge-Based Systems), Annecy in France, (ESIA-Université de Savoie, France, July 2002) 1, 463-469.

18. Y.Yoshida, The valuation of European options in uncertain environment, European J. Oper. Res. 145 (2003) 221-229.

19. L.A.Zadeh, Fuzzy sets, Inform. and Control 8 (1965) 338-353.

20. Z.Zmeškal, Application of the fuzzy-stochastic methodology to appraising the firm value as a European call option, European J. Oper. Res. 135 (2001) 303310.

Address for Offprints: Y.Yoshida

Faculty of Economics and Business Administration,

The University of Kitakyushu, Kokuraminami,

Kitakyushu 802-8577, Japan. 
DiscAmerOption3b.tex; 31/08/2005; $10: 49 ;$ p.18 